\title{
AN ORDER PROPERTY FOR FAMILIES OF SETS
}

\author{
N. H. WILLIAMS
}

(Received 17 June 1986)

Communicated by C. J. Ash

\begin{abstract}
We develop the idea of a $\theta$-ordering (where $\theta$ is an infinite cardinal) for a family of infinite sets. A $\theta$-ordering of the family $A$ is a well ordering of $A$ which decomposes $A$ into a union of pairwise disjoint intervals in a special way, which facilitates certain transfinite constructions. We show that several standard combinatorial properties, for instance that of the family $A$ having a $\theta$-transversal, are simple consequences of $A$ possessing a $\theta$-ordering. Most of the paper is devoted to showing that under suitable restrictions, an almost disjoint family will have a $\theta$-ordering. The restrictions involve either intersection conditions on $A$ (the intersection of every $\lambda$-size subfamily of $A$ has size at most $\kappa$ ) or a chain condition on $A$.
\end{abstract}

1980 Mathematics subject classification (Amer. Math. Soc.): 03 E 05, 04 A 20.

\section{Introduction}

The family of sets $A$ is said to be a $(\lambda, \kappa)$-family if $|A|=\lambda$ and $|A|=\kappa$ for all $A$ in $A$. The family $A$ is said to be almost disjoint if $|A \cap B|<|A|,|B|$ for all distinct, $A, B$ in $A$. Our interest in this paper is in almost disjoint $(\lambda, \kappa)$-families $A$ which possess what we call a $\theta$-ordering, for various values of $\theta$ with $\theta \leq \kappa$.

DEFINITION 1.1. A $\theta$-ordering of the $(\lambda, \kappa)$-family $A$ is a (strict) well order $\prec$ of $A$ under which there is a family $I$ of pairwise disjoint intervals with $A=\bigcup I$ such that $|I| \leq \kappa$ for each $I \in I$, and for each $I \in I$ and $A \in A$ :

$$
A \in I \Rightarrow \mid A \cap \bigcup\{\bigcup J ; J \in I \text { and } J \prec I\} \mid<\theta,
$$

(C) 1988 Australian Mathematical Society $0263-6115 / 88 \$ A 2.00+0.00$ 


$$
A \in I \Rightarrow|A \cap \bigcup\{B \in I ; B \prec A\}|<\kappa,
$$

(where in (1), $J \prec I$ means that $B \prec A$ for all $A \in I$ and $B \in J$ ).

A $(\lambda, \kappa)$-family clearly has a $\theta$-ordering if $\theta>\kappa$ and so we always assume $\theta \leq \kappa$. Notice that a $\kappa$-ordering of the $(\lambda, \kappa)$-family $A$ is just a well ordering $\prec$ of $A$ such that for each $A \in \mathcal{A}$,

$$
|A \cap \bigcup\{B \in A ; B \prec A\}|<\kappa,
$$

for we can take each interval in the family $I$ to be a singleton set. The special case of an $\aleph_{0}$-ordering of a family of denumerable sets has appeared previously, see Davies and Erdös ([1], Lemma 3).

Obviously, any $(\lambda, \kappa)$-family which has a $\theta$-ordering where $\theta \leq \kappa$ must be almost disjoint. Any almost disjoint $(\kappa, \kappa)$-family $A$ where $\kappa$ is regular has a $\theta$ ordering, for any $\theta \leq \kappa$, since any well ordering of $A$ of order type $\kappa$ with $A$ itself the only interval gives a $\theta$-ordering. However, not every almost disjoint $\left(\kappa^{+}, \kappa\right)$ family possesses even a $\kappa$-ordering, for let $S$ be a $(\kappa, \kappa)$-family of pairwise disjoint sets, and let $A$ with $S \subseteq A$ be an almost disjoint $\left(\kappa^{+}, \kappa\right)$-family with $\bigcup A=\bigcup S$. Any $A \in A$ coming after all the sets in $S$ in a well ordering $\prec$ of $A$ has $A \cap$ $\bigcup\{B \in A ; B \prec A\}=A$, so $A$ has no $\kappa$-ordering. If $\kappa$ is singular, there are almost disjoint $(\kappa, \kappa)$-families that are maximal with respect to almost disjointness (see Erdös and Hechler [3]). No such maximal family possesses even a $\kappa$-ordering (for if $\prec$ is a $\kappa$-ordering of the $(\kappa, \kappa)$-family $A$, choosing $x(A) \in A-\bigcup\{B ; B \prec A\}$ and putting $T=\{x(A) ; A \in A\}$ gives a set almost disjoint from each member of $A)$. If $\kappa$ is singular, to ensure that the $(\kappa, \kappa)$-family $A$ has a $\theta$-ordering we need to assume the stronger condition that always $|A \cap B|<\eta$ for some fixed $\eta<\kappa$.

Before we explain our interest in $\theta$-orderings, we need some more terminology. The family $A$ is said to satisfy the intersection condition $C(\eta, \theta)$ if $|\cap B|<\theta$ for all subfamilies $B$ of $A$ with $|B|=\eta$. A set $T$ is called a $\theta$-transversal of the family $A$ if $1 \leq|T \cap A|<\theta$ for all $A$ in $A$. The family $A$ is said to be sparse if there is a function $f: A \rightarrow P \bigcup A$ with $f(A) \subseteq A$ and $|f(A)|<|A|$ for all $A$ in $A$, such that $\{A-f(A) ; A \in A\}$ is a pairwise disjoint family.

It is a theorem of Erdös and Hajnal ([2], Theorem 7) that every $(\lambda, \kappa)$-family satisfying $C(2, \theta)$ has a $\theta^{+}$-transversal, provided $\lambda$ is not too large (and with some restriction on $\theta<\kappa)$. It was recently shown by Komjáth ([5], Theorem 5 ) that, under similar conditions, every such family is sparse. The proofs of these two results are little involved. A similar inductive construction is used in both cases, though the details are different. Our interest in $\theta$-orderings was aroused by the observation that the families in question possess a $\theta^{+}$-order, and it is almost trivial to deduce from this that they are sparse and have a $\theta^{+}$ transversal. Several further properties we looked at turned out to be an easy consequence of a $\theta$-ordering. For instance, in ([8], Theorem 3.2) we showed that 
provided $\lambda$ is not too large, every almost disjoint $(\lambda, \kappa)$-family satisfying certain chain conditions has a $\kappa$-transversal. We shall show in Section 3 that under these circumstances, the family possesses a $\kappa$-ordering. The existence of a $\kappa$ transversal follows easily from this. Here, by a chain condition we mean the following. The family $\mathcal{A}$ satisfies the $\mu$-chain condition if there is no set $D \subseteq \cup \mathcal{A}$ and sequence $\left(A_{\alpha} ; \alpha<\mu\right)$ of sets from $A$ such that $D \cap A_{\alpha} \subset D \cap A_{\beta}$ whenever $\alpha<\beta<\mu$ (where $\subset$ means strict inclusion).

The main results which we prove concerning the existence of $\theta$-orderings are the following. (These results appear in Theorems 2.4, 2.6, 3.3 and 3.4 below.)

THEOREM 1.2. Let $A$ be an almost disjoint $(\lambda, \kappa)$-family.

(a) $(G C H)$ If $\theta^{+}<\kappa$ and either $A$ satisfies $C(2, \theta)$, or else $\kappa$ is regular and $A$ satisfies $C\left(\kappa^{+}, \theta\right)$, then $A$ has a $\theta^{++}$-ordering.

(b) $(G C H)$ If $\theta^{+} \leq \kappa$ and $c f(\mu) \neq c f(\theta)$ whenever $\kappa \leq \mu<\lambda$, and $A$ satisfies the same intersection conditions as in (a), then $A$ has a $\theta^{+}$-ordering.

(c) $(V=L)$ If $\theta^{+} \leq \kappa$ and $A$ satisfies the same intersection conditions as in (a), then $A$ has a $\theta^{+}$-ordering.

(d) If $\kappa$ is regular and $A$ satisfies the $\aleph_{0}$-chain condition, then $A$ has a $\kappa$ ordering.

(e) $(G C H)$ If $\kappa$ is regular, and $c f(\mu) \neq \aleph_{0}$ whenever $\kappa<\mu<\lambda$, and $A$ satisfies the $\kappa$-chain condition, then $A$ has a $\kappa$-ordering.

(f) $(V=L)$ If $\kappa$ is regular and $A$ satisfies the $\kappa$-chain condition then $A$ has a $\kappa$-ordering.

The paper is organized as follows. We continue this introduction with a couple of simple observations that provide our constructions for $\theta$-orderings. In Section 2 we give the existence proofs when the family $A$ satisfies the intersection conditions. Section 3 is devoted to the results under the chain conditions. And in Section 4 we give a number of applications.

Our notation is mostly standard. We use $[A]^{\eta}$ or $[A]^{<\eta}$ for the set of all subsets $B$ of $A$ with $|B|=\eta$ or $|B|<\eta$, respectively, and ${ }^{<\eta} A$ for the set of all sequences of elements of $A$ of length less than $\eta$. Weak cardinal exponentiation is indicated by $\kappa^{<\lambda}$. The cofinality of the cardinal $\kappa$ is written $c f(\kappa)$. The cardinal successor of $\kappa$ is $\kappa^{+}$, and $\kappa^{\alpha+}$ is the iteration of this $\alpha$ times. For cardinal $\kappa$, by a $\kappa$-sequence we mean a non-decreasing sequence $\left(\kappa_{\sigma} ; \sigma<c f(\kappa)\right)$ of cardinals $\kappa_{\sigma}<\kappa$ with $\kappa=\sum\left(\kappa_{\sigma} ; \sigma<c f(\kappa)\right)$. The letters $\eta, \theta, \kappa, \lambda, \mu$ will be used for infinite cardinal numbers, and other lower case Greek letters for ordinal numbers (with $\omega$ for the least infinite ordinal). The letters $i, m, n$ will be used for finite ordinals.

We conclude this section with two constructions that will be used in the following sections. 
LEMMA 1.3. Let $\theta^{+} \leq \kappa$. Suppose the $(\lambda, \kappa)$-family $A$ satisfies

(i) every $B$ in $[A]^{<\lambda}$ has a $\theta^{+}$-ordering, and

(ii) for every $C \in[A]^{<\lambda}$ there is $C^{*} \in[A]^{<\lambda}$ with $C \subseteq C^{*}$ such that

$$
\forall A \in A\left(\left|A \cap \bigcup C^{*}\right| \geq \theta \Rightarrow A \in C^{*}\right) \text {. }
$$

Then $A$ possesses a $\theta^{+}$-ordering.

Proof. Write $A=\bigcup\left\{A_{\sigma} ; \sigma<c f(\lambda)\right\}$ where always $\left|A_{\sigma}\right|<\lambda$. Define recursively $B_{\sigma} \in[A]<\lambda$ for $\sigma<c f(\lambda)$ by

$$
B_{\sigma}=\left(A_{\sigma} \cup \bigcup\left\{B_{\tau} ; \tau<\sigma\right\}\right)^{*} .
$$

Then $A=\bigcup\left\{B_{\sigma} ; \sigma<c f(\lambda)\right\}$, and for each $A \in A$ let $\sigma(A)$ be the least $\sigma$ such that $A \in B_{\sigma}$. Thus if $\tau<\sigma(A)$ then $A \notin B_{\tau}$ so $\left|A \cap \bigcup B_{\tau}\right|<\theta$ by (4), since $B_{\tau}=C^{*}$ for $C=A_{\sigma} \cup \bigcup\left\{B_{\tau} ; \tau<\sigma\right\}$.

Hence

$$
\left|A \cap \bigcup\left\{\bigcup B_{\tau} ; \tau<\sigma(A)\right\}\right|<\theta^{+},
$$

since $\bigcup B_{\rho} \subseteq \bigcup B_{\tau}$ for $\rho<\tau<\sigma(A)$.

By (i), there is a $\theta^{+}$-ordering of $B_{\sigma}$, say $\prec_{\sigma}$ with family of intervals $I_{\sigma}$. Define $\prec$ on $A$ by:

$$
A \prec B \Leftrightarrow \sigma(A)<\sigma(B) \text { or }\left[\sigma(A)=\sigma(B) \text { and } A \prec_{\sigma(A)} B\right] .
$$

Clearly this is a well order of $A$, and for each $I \in I_{\sigma}$, if $I^{*}=I-\bigcup\left\{\bigcup B_{r} ; \tau<\sigma\right\}$ then $I^{*}$ is an interval (possibly empty) of $\prec$. Put $I=\left\{I^{*} ; \exists \sigma<c f(\lambda)\left(I \in I_{\sigma}\right)\right\}$, so $A=\bigcup I$. Take any $A \in A$. If $A \in K$ for some $K \in I$, then there must be $I \in$ $I_{\sigma(A)}$ with $K=I^{*}$ and $A \in I$. Since $\{B \in K ; B \prec A\} \subseteq\left\{B \in I ; B \prec_{\sigma(A)} A\right\}$, certainly $|A \cap \bigcup\{B \in K ; B \prec A\}|<\kappa$ since $\prec_{\sigma(A)}$ is a $\theta^{+}$-ordering. To establish that $\prec$ is a $\theta^{+}=$ordering of $A$, it remains to show that

$$
\mid A \cap \bigcup\{\bigcup L ; L \in I \text { and } L \prec K\} \mid<\theta^{+} \text {. }
$$

Take $L \in I$ with $L \prec K$. The $L=J^{*}$ for some $J$ where either $J \in I_{\tau}$ for some $\tau<\sigma(A)$, or $J \in I_{\sigma(A)}$ with $J \prec_{\sigma(A)} I$. Since

$$
\bigcup\left\{\bigcup J^{*} ; J \in I_{\sigma(A)} \text { and } J \prec_{\sigma(A)} I\right\} \subseteq \bigcup\left\{\bigcup J ; J \in I_{\sigma(A)} \text { and } J \prec_{\sigma(A)} I\right\}
$$

and $\prec_{\sigma(A)}$ is a $\theta^{+}$-ordering we have $\mid A \cap \bigcup\left\{\bigcup J^{*} ; J \in I_{\sigma(A)}\right.$ and $\left.J \prec_{\sigma(A)} I\right\} \mid<$ $\theta^{+}$. Also $\bigcup\left\{\bigcup J^{*} ; J \in I_{\tau}\right.$ and $\left.\tau<\sigma(A)\right\} \subseteq \bigcup\left\{\bigcup B_{\tau} ; \tau<\sigma(A)\right\}$ so by (5), $\mid A \cap \bigcup\left\{\bigcup J^{*} ; J \in I_{\tau}\right.$ and $\left.\tau<\sigma(A)\right\} \mid<\theta^{+}$. Hence (6) holds, and so $\prec$ is a $\theta^{+}$-ordering of $A$. 
LEMMA 1.4. Let $c f(\lambda)>\omega$. Suppose the $(\lambda, \kappa)$-family $A$ satisfies

(i) every $B$ in $[A]^{<\lambda}$ has $\theta$-ordering, and

(ii) there is a family $\left\{U_{\rho} ; \rho<c f(\lambda\}\right.$ with $\bigcup \mathcal{A}=\bigcup\left\{U_{\rho} ; \rho<c f(\lambda)\right\}$ and $U_{\rho} \subseteq U_{\tau}$ whenever $\rho<\tau<c f(\lambda)$, such that

(a) $\forall A \in A \exists \rho<c f(\lambda) \exists n \geq 1\left(A \subseteq U_{\rho+n}\right.$ and $\left.\left|A \cap U_{\rho}\right|<\theta\right)$,

(b) $\forall \rho<c f(\lambda)\left(\left|\left\{A \in A ; A \subseteq U_{\rho}\right\}\right|<\lambda\right)$.

Then $A$ possesses a $\theta$-ordering.

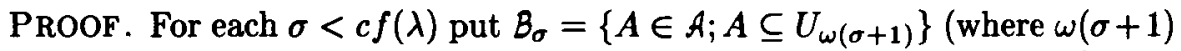
means the ordinal product), so $B_{\sigma} \subseteq B_{\tau}$ if $\sigma<\tau<c f(\lambda)$, and $\left.B_{\sigma} \in[A]\right]^{<\lambda}$ with $A=\bigcup\left\{B_{\sigma} ; \sigma<c f(\lambda)\right\}$. For each $A \in A$ let $\sigma(A)$ be the least $\sigma$ such that $A \in B_{\sigma}$. Then by (a), $A \subseteq U_{\omega \sigma+m}$ for some $m \geq 1$, and $\left|A \cap U_{\omega \sigma(A)}\right|<\theta$. In particular, since

$$
\bigcup\left\{\bigcup B_{\tau} ; \tau<\sigma(A)\right\}=\bigcup\left\{U_{\omega(\tau+1)} ; \tau<\sigma(A)\right\} \subseteq U_{\omega \sigma(A)}
$$

we have $\left|A \cap \bigcup\left\{\bigcup B_{\tau} ; \tau<\sigma(A)\right\}\right|<\theta$. By (i), there is a $\theta$-ordering $\prec_{\sigma}$ of $B_{\sigma}$. Define $\prec$ on $A$ by

$$
A \prec B \Leftrightarrow \sigma(A)<\sigma(B) \text { or }\left[\sigma(A)=\sigma(B) \text { and } A \prec_{\sigma(A)} B\right] .
$$

Then just as in the proof of Lemma $1.3, \prec$ is a $\theta$-ordering of $A$.

\section{Intersection conditions}

We shall make use of the following result, going back to Tarski (for example, see ([6], Lemma 3.2.3 and Corollary 3.2.4)).

LEMMA $2.1(G C H)$. Suppose $|S|=\mu$ and let $A$ be a family of subsets of $S$ satsifying $C\left(\mu^{+}, \theta\right)$. Then $|A| \leq \mu$ provided either

$$
\begin{gathered}
\theta^{+} \leq \mu \text { and } \forall A \in A(|A|>\theta) \text {, or } \\
\theta \leq \mu \text { and } c f(\theta) \neq c f(\mu) \text { and } \forall A \in \mathcal{A}(|A| \geq \theta) \text {. }
\end{gathered}
$$

The following two lemmas, combined with Lemma 1.3, will enable us to prove parts (a) and (b) of Theorem 1.2.

LEMMA $2.2(G C H)$. Let $\theta^{+} \leq \kappa$. Suppose the $(\lambda, \kappa)$-family $A$ satisfies $C\left(\kappa^{+}, \theta\right)$. Then for each $C \in[A] \geq \kappa$ there is $C^{*} \subseteq \mathcal{A}$ with $C \subseteq C^{*}$ and $\left|C^{*}\right|=|C|$ such that

$$
\forall A \in A\left(\left|A \cap \bigcup C^{*}\right| \geq \theta^{+} \Rightarrow A \in C^{*}\right) .
$$


ProOF. Take $C \in[A] \geq \kappa$. Recursively define families $C_{i}$ for $i<\omega$ by putting $C_{0}=C$ and $C_{i+1}=\left\{A \in A ;\left|A \cap \bigcup C_{i}\right|>\theta\right\}$. Define $C^{*}=\bigcup\left\{C_{i} ; i<\omega\right\}$. Always $C_{i} \subseteq C_{i+1}$ so $\left|C_{i}\right| \geq \kappa$ and hence $\left|\bigcup C_{i}\right|=\left|C_{i}\right|$. Lemma 2.1 ensures that $\left|\left\{A \cap \bigcup C_{i} ; A \in C_{i+1}\right\}\right| \leq\left|\bigcup C_{i}\right|=\left|C_{i}\right|$. For each $X \in\left[\cup C_{i}\right]^{>\theta}$ we have $\left|\left\{A \in A ; ; A \cap \bigcup C_{i}=X\right\}\right| \leq \kappa$ since $A$ satisfies $C\left(\kappa^{+}, \theta\right)$. Hence $\left|C_{i+1}\right| \leq \kappa \times\left|C_{i}\right|=$ $\left|C_{i}\right|$ so that $\left|C_{i+1}\right|=\left|C_{i}\right|$, for all $i<\omega$. Thus $\left|C^{*}\right|=\left|C_{0}\right|=|C|$. Also if we have $A \in A$ with $\left|A \cap \bigcup C^{*}\right|>\theta$, then since $\bigcup C^{*}=\bigcup\left\{\bigcup C_{i} ; i<\omega\right\}$, we must have $\left|A \cap \bigcup C_{i}\right|>\theta$ for some $i<\omega$, so that $A \in C_{i+1}$ and thus $A \in C^{*}$. Thus $C^{*}$ has the required properties.

LEMMA $2.3(G C H)$. Suppose $\theta^{+} \leq \kappa<\lambda$ and $\lambda \neq \mu^{+}$where $c f(\theta)=c f(\mu)$. Suppose the $(\lambda, \kappa)$-family $A$ satisfies $C\left(\kappa^{+}, \theta\right)$. Then for each $C \in[A]<\lambda$ there is $C^{*} \in[A]^{<\lambda}$ with $C \subseteq C^{*}$ such that

$$
\forall A \in A\left(\left|A \cap \bigcup C^{*}\right| \geq \theta \Rightarrow A \in C^{*}\right) .
$$

ProOF. Suppose first $c f(\theta) \neq \omega$. This case is similar to the proof of the previous lemma. Take $C \in[A]^{<\lambda}$, and we may suppose $|C| \geq \kappa$. Recursively define families $C_{i}$ for $i<\omega$ by putting $C_{0}=C$ and $C_{i+1}=\left\{A \in A ;\left|A \cap \bigcup C_{i}\right| \geq \theta\right\}$, and put $C^{*}=\bigcup\left\{C_{i} ; i<\omega\right\}$. As before, $\left|\bigcup C_{i}\right|=\left|C_{i}\right|$. Let $|C|=\mu$. We show by induction that always $\left|C_{i}\right|=\mu$ if $c f(\mu) \neq c f(\theta)$, and always $\left|C_{i}\right| \leq \mu^{+}$if $c f(\mu)=c f(\theta)$. Put $D_{i}=\left\{A \cap \bigcup C_{i} ; A \in C_{i+1}\right\}$. Suppose $c f(\mu) \neq c f(\theta)$, and $\left|C_{i}\right|=\mu$. Then $\left|D_{i}\right| \leq \mu$ by Lemma 2.1. Since $A$ satisfies $C\left(\kappa^{+}, \theta\right)$, as in the proof of Lemma 2.2, this ensures that $\left|C_{i+1}\right|=\mu$. Now suppose $c f(\mu)=c f(\theta)$. If $\left|C_{i}\right|=\mu^{+}$, since $c f\left(\mu^{+}\right) \neq c f(\theta)$, just as above this ensures that $\left|C_{i+1}\right|=\mu^{+}$. Whereas if $\left|C_{i}\right|=\mu$, since $D_{i} \subseteq\left[\cup C_{i}\right]^{\geq \theta}$ certainly $\left|D_{i}\right| \leq \mu^{+}$so still $\left|C_{i+1}\right| \leq \mu^{+}$. This completes the induction. Now $C^{*}=\bigcup\left\{C_{i} ; i<\omega\right\}$, so $\left|C^{*}\right|=\mu<\lambda$ if $c f(\mu) \neq c f(\theta)$. If $c f(\mu)=c f(\theta)$ then $\left|C^{*}\right| \leq \mu^{+}$, and by hypothesis in this case $\mu^{+} \neq \lambda$, so still $\left|C^{*}\right|<\lambda$. To see that (10) holds, take any $A$ in $A$ with $\left|A \cap \bigcup C^{*}\right| \geq \theta$. Since $\bigcup C^{*}=\bigcup\left\{\bigcup \mathcal{C}_{i} ; i<\omega\right\}$ and $c f(\theta) \neq \omega$, we must have $\left|A \cap \bigcup C_{i}\right| \geq \theta$ for some $i>\omega$, so that $A \in C_{i+1}$, and hence $A \in C^{*}$.

Now consider the case $c f(\theta)=\omega$. Take $C \in[\mathcal{A}]^{\mu}$ where we may assume $\kappa \leq \mu<\lambda$. This time we recursively define families $C_{\alpha}$ for $\alpha<\omega_{1}$ by putting $C_{0}=C, C_{\alpha+1}=\left\{A \in A ;\left|A \cap \bigcup C_{\alpha}\right| \geq \theta\right\}$, and $C_{\alpha}=\bigcup\left\{C_{\beta} ; \beta<\alpha\right\}$ when $\alpha$ is a limit ordinal. Put $C^{*}=\bigcup\left\{C_{\alpha} ; \alpha<\omega_{1}\right\}$. Similarly to the previous case, we show by induction that always $\left|C_{\alpha}\right|=\mu$ if $c f(\mu) \neq c f(\theta)$ or $\left|\mathcal{C}_{\alpha}\right| \leq \mu^{+}$if $c f(\mu)=c f(\theta)$. If follows that $\left|C^{*}\right|<\lambda$ (noting that $\lambda>\aleph_{1}$, for this case to hold). To see that (10) holds, take any $A \in A$ with $\left|A \cap \bigcup \mathcal{C}^{*}\right| \geq \theta$. Still $\bigcup \mathcal{C}^{*}=\bigcup\left\{\bigcup \mathcal{C}_{\alpha} ; \alpha<\omega_{1}\right\}$. Take an increasing $\theta$-sequence $\left(\theta_{n} ; n<\omega\right)$. For each $n$ there is $\alpha_{n}<\omega_{1}$ such that $\left|A \cap \bigcup\left\{\bigcup C_{\alpha} ; \alpha<\alpha_{n}\right\}\right| \geq \theta_{n}$. Let $\beta$ be the least limit ordinal larger than all the $\alpha_{n}$, so $\beta<\omega_{1}$ and $\left|A \cap \bigcup\left\{\bigcup \mathcal{C}_{\alpha} ; \alpha<\beta\right\}\right| \geq \theta$; thus $\left|A \cap \bigcup \mathcal{C}_{\beta}\right| \geq \theta$ so $A \in C_{\beta+1}$ and hence $A \in C^{*}$. 
THEOREM $2.4(G C H)$. Let $A$ be an almost disjoint $(\lambda, \kappa)$-family. Suppose either $A$ satisfies $C(2, \theta)$, or else $\kappa$ is regular and $A$ satisfies $C\left(\kappa^{+}, \theta\right)$.

(a) If $\theta^{+}<\kappa$, then $A$ has a $\theta^{++}$-ordering.

(b) If $\theta^{+} \leq \kappa$, and $(\kappa \leq \mu<\lambda \Rightarrow c f(\mu) \neq c f(\theta))$, then $A$ possesses a $\theta^{+}$-ordering.

PrOOF. For $\lambda \leq \kappa$, if $A$ satisfies $C(2, \theta)$ any well order of $A$ of order type $\lambda$ is suitable, and the same is true if $\kappa$ is regular since $A$ is almost disjoint. For $\lambda>\kappa$ we proceed by induction on $\lambda$. Take a suitable $(\lambda, \kappa)$-family $A$. Consider (b) first. We shall use Lemma 1.3. By the inductive hypothesis, (i) of Lemma 1.3 holds, and (ii) holds by Lemma 2.3, noting that under the conditions in (b), $\lambda \neq \mu^{+}$ with $c f(\mu)=c f(\theta)$. So Lemma 1.3 ensures that $A$ possesses a $\theta^{+}$-ordering.

Similarly for (a), Lemmas 2.2 and 1.3 show that $A$ has a $\theta^{++}$-ordering.

The least $\lambda$ for which (b) in Lemma 2.4 does not apply is when $\lambda=\kappa^{c f(\theta)+}$. This method of proof fails for larger $\lambda$, though the result may still be true. Indeed, under stronger set theoretic hypotheses the restriction on $\lambda$ may be lifted. We can continue the transfinite induction if we assume Jensen's principle $\square_{\mu}$ whenever $c f(\mu)=c f(\theta)$. It is well known that if the axiom of constructibility $(V=L)$ is assumed, then $\square_{\mu}$ holds for all $\mu$. The statement $\square_{\mu}$ asserts: for each limit ordinal $\alpha<\mu^{+}$there is a closed unbounded set $C_{\alpha} \subseteq \alpha$ such that $\left|C_{\alpha}\right|<\mu$ whenever $c f(\alpha)<\mu$, and $C_{\beta}=C_{\alpha} \cap \beta$ whenever $\beta$ is a limit point of $C_{\alpha}$. It is convenient first to isolate the construction from $\square_{\mu}$ that we require.

LEMMA 2.5. Suppose $\mu$ is singular, and assume $\square_{\mu}$. For each limit ordinal $\alpha<\mu^{+}$there is a decomposition $\alpha=\bigcup\{T(\alpha, \sigma) ; \sigma<c f(\mu)\}$ where each $T(\alpha, \sigma)$ is cofinal in $\alpha$ with $|T(\alpha, \sigma)|<\mu$ and there is a subset $D(\alpha) \subseteq \alpha$ with $|D(\alpha)|<\mu$ such that $T(\gamma, \sigma) \subseteq T(\beta, \sigma)$ whenever $\beta, \gamma \in D(\alpha)$ with $\gamma<\beta$. If $c f(\alpha)>\omega$ then $D(\alpha)$ is cofinal in $\alpha$ and $T(\alpha, \sigma)=\bigcup\{T(\beta, \sigma) ; \beta \in D(\alpha)\}$.

ProOF. (See Komjáth [5].) Let $\left(\mu_{\sigma} ; \sigma<c f(\mu)\right)$ be an increasing $\mu$-sequence. For each limit $\alpha<\mu^{+}$, take sets $C_{\alpha}$ as provided by $\square_{\mu}$ where we may suppose $0 \in C_{\alpha}$, and let $\left(c_{\alpha \xi} ; \xi<\right.$ ot $\left.\left(C_{\alpha}\right)\right)$ be the increasing enumeration of $C_{\alpha}$, where ot $\left(C_{\alpha}\right)$ means the order type of $C_{\alpha}$. Put $D(\alpha)=\left\{\beta \in C_{\alpha} ; \beta\right.$ is a limit point of $\left.C_{\alpha}\right\}$. For $\beta \in D(\alpha)$ we have $C_{\beta}=C_{\alpha} \cap \beta$ so $c_{\beta \xi}=c_{\alpha \xi}$ whenever $\xi<$ ot $\left(C_{\beta}\right)$. For $\gamma<\delta<\mu^{+}$, fix a decomposition $\{\xi ; \gamma \leq \xi<\delta\}=\bigcup\{S(\gamma, \delta, \sigma) ; \sigma<c f(\mu)\}$ where always $1 \leq|S(\gamma, \delta, \sigma)| \leq \mu_{\sigma}$. For $\sigma<c f(\mu)$ and limit $\alpha<\mu^{+}$, put $T(\alpha, \sigma)=\bigcup\left\{S\left(c_{\alpha \xi}, c_{\alpha \xi+1}, \sigma\right) ; \xi<\right.$ ot $\left.\left(C_{\alpha}\right)\right\}$, so $\alpha=\bigcup\{T(\alpha, \sigma) ; \sigma<c f(\mu)\}$. Whenever $\beta \in D(\alpha)$ we have $T(\beta, \sigma)=T(\alpha, \sigma) \cap \beta$. Hence $T(\gamma, \sigma) \subseteq T(\beta, \sigma)$ if $\beta, \gamma \in D(\alpha)$ with $\gamma<\beta$. If $c f(\alpha)>\omega$, then $D(\alpha)$ is cofinal in $\alpha$, and so $T(\alpha, \sigma)=\bigcup\{T(\beta, \sigma) ; \beta \in D(\alpha)\}$. Also $|D(\alpha)| \leq\left|C_{\alpha}\right|$ and $\left|C_{\alpha}\right|<\mu$ since $\mu$ is singular. Finally $|T(\alpha, \sigma)| \leq \sum\left(\left|S\left(c_{\alpha \xi}, c_{\alpha \xi+1}, \sigma\right)\right| ; \xi<\right.$ ot $\left.\left(C_{\alpha}\right)\right) \leq \mu_{\sigma} \times\left|C_{\alpha}\right|<\mu$. 
THEOREM 2.6 $(V=L)$. Suppose $\theta^{+} \leq \kappa$. Let $\mathcal{A}$ be an almost disjoint $(\lambda, \kappa)$ family satisfying either $C(2, \theta)$, or, if $\kappa$ is regular, $C\left(\kappa^{+}, \theta\right)$. Then $A$ possesses a $\theta^{+}$-ordering.

Proof. As in the proof of Theorem 2.4(b), we may suppose $\lambda>\kappa$ and proceed by induction on $\lambda$. The previous argument holds unless $\lambda=\mu^{+}$where $c f(\mu)=c f(\theta)$. So suppose indeed that $\lambda=\mu^{+}$, with $c f(\mu)=c f(\theta)$. Let $\mathcal{A}=\left\{A_{\delta} ; \delta<\mu^{+}\right\}$, and we may suppose $\bigcup \mathcal{A}=\mu^{+}$. We have the sets $D(\alpha)$ and $T(\alpha, \sigma)$ for $\alpha<\mu^{+}$and $\sigma<c f(\mu)$, as in Lemma 2.5.

Define limit ordinals $l_{\varepsilon}<\mu^{+}$by transfinite recursion for $\varepsilon<\mu^{+}$as follows. Let $l_{0}$ be the least limit ordinal $\gamma>\bigcup A_{0}$, and for $\varepsilon$ a limit ordinal, put $l_{\varepsilon}=$ $\bigcup\left\{l_{\delta} ; \delta<\varepsilon\right\}$. At successor stages, put

$$
B_{\varepsilon}=\left\{A \in A ; ; \exists \text { limit } \alpha<l_{\varepsilon} \exists \sigma<c f(\mu)(|A \cap T(\alpha, \sigma)| \geq \theta)\right\},
$$

and define $l_{\varepsilon+1}$ to be the least ordinal $\gamma>l_{\varepsilon} \cup \bigcup A_{\varepsilon} \cup \bigcup \cup B_{\varepsilon}$ with $c f(\gamma)>\omega$. To see that then $l_{\varepsilon+1}<\mu^{+}$, note that since $|T(\alpha, \sigma)|<\mu$ we have $\left|[T(\alpha, \sigma)]^{\theta}\right| \leq \mu$, and for each $X \in[T(\alpha, \sigma)]^{\theta}$ since $A$ satisfies $C\left(\kappa^{+}, \theta\right)$ we have $\mid\{A \in A ; A \cap$ $T(\alpha, \sigma)=X\} \mid \leq \kappa$, so that $\left|\beta_{\varepsilon}\right| \leq \mu$ and hence $l_{\varepsilon+1}<\mu^{+}$.

Take $A \in A$, and we show by induction on $\varepsilon<\mu^{+}$that if $\left|A \cap l_{\varepsilon}\right| \geq \theta^{+}$then $A \in B_{\varepsilon}$. If $c f\left(l_{\varepsilon}\right)>\omega$, by Lemma 2.5, $l_{\varepsilon}=\bigcup\left\{T\left(l_{\varepsilon}, \sigma\right) ; \sigma<c f(\mu)\right\}$ so there must be $\sigma<c f(\mu)$ such that $\left|A \cap T\left(l_{\varepsilon}, \sigma\right)\right| \geq \theta^{+}$, since $c f(\mu)=c f(\theta)<\theta^{+}$. Now $T\left(l_{\varepsilon}, \sigma\right)=\bigcup\left\{T(\beta, \sigma) ; \beta \in D\left(l_{\varepsilon}\right)\right\}$ and $T(\gamma, \sigma) \subseteq T(\beta, \sigma)$ for $\beta, \gamma \in D\left(l_{\varepsilon}\right)$ with $\gamma<\beta$, so there must be $\beta \in D\left(l_{\varepsilon}\right)$ such that $|A \cap T(\beta, \sigma)| \geq \theta$. And $\beta \in D\left(l_{\varepsilon}\right) \subseteq l_{\varepsilon}$, so $A \in B_{\varepsilon}$ as claimed. If $c f\left(l_{\varepsilon}\right)=\omega$ there are $\varepsilon(n)<\varepsilon$ such that $l_{\varepsilon}=\bigcup\left\{l_{\varepsilon(n)} ; n<\omega\right\}$ and since $\left|A \cap l_{\varepsilon}\right| \geq \theta^{+}$there must be $n<\omega$ with $\left|A \cap l_{\varepsilon(n)}\right| \geq \theta^{+}$. Then the inductive hypothesis gives that $A \in B_{\varepsilon(n)}$, so $A \in B_{\varepsilon}$.

For $A \in \mathcal{A}$, define $\varepsilon(A)$ to be the least $\varepsilon$ such that $A \in B_{\varepsilon}$. (Such $\varepsilon(A)$ exists, for if $A=A_{\delta}$ we have $A \subseteq l_{\delta+1}$, so $A \in B_{\delta+1}$ by the previous observation.) Now $\varepsilon(A)$ is not a limit ordinal, since for limit $\varepsilon$ we have $l_{\varepsilon}=\bigcup\left\{l_{\delta} ; \delta<\varepsilon\right\}$ so $B_{\varepsilon}=\bigcup\left\{B_{\delta}: \delta<\varepsilon\right\}$. And if $\rho<\varepsilon(A)$ we must have $\left|A \cap l_{\rho}\right|<\theta^{+}$, for if $\left|A \cap l_{\rho}\right| \geq \theta^{+}$then $A \in B_{\rho}$ by the observation above. Also since $A \in B_{\varepsilon(A)}$ and UU $B_{\varepsilon(A)} \subseteq l_{\varepsilon(A)+1}$ we have $A \subseteq l_{\varepsilon(A)+1}$.

We show that the conditions of Lemma 1.4 are satsified for $A$ to possess a $\theta^{+}$-ordering. Every $B$ in $[A]^{<\lambda}$ has a $\theta^{+}$-ordering by the inductive hypothesis, so (i) of Lemma 1.4 holds. For (ii), define $U_{\rho}=l_{\rho}$, for $\rho<\mu^{+}=c f(\lambda)$. Certainly $\bigcup A=\mu^{+}=\bigcup\left\{U_{\rho} ; \rho<\mu^{+}\right\}$and $U_{\rho} \subseteq U_{\tau}$ if $\rho<\tau<\mu^{+}$. Take any $A \in A$. Since $\varepsilon(A)$ is not a limit ordinal, we can put $\rho=\varepsilon(A)-1$. Since $A \subseteq l_{\varepsilon(A)+1}$, we have $A \subseteq U_{\rho+2}$, and since $\rho<\varepsilon(A)$ we have $\left|A \cap U_{\rho}\right|<\theta^{+}$. Hence (iia) holds. For 
(iib), suppose $\rho<\mu^{+}$is given. If $A \subseteq U_{\rho}=l_{\rho}$ then $A \in B_{\rho}$, but $\left|B_{\rho}\right| \leq \mu$ and so (iib) is satisfied. Hence by Lemma $1.4, A$ possesses a $\theta^{+}$-ordering.

\section{Chain conditions}

We shall need the following lemma, from Williams [8].

LEMMA 3.1. Let $\kappa$ be regular and suppose $A$ is an almost disjoint $(\lambda, \kappa)$ family which satisfies either

(a) the $\aleph_{0}$-chain condition, or

(b) $(G C H)$ the $\kappa$-chain condition.

Then $\lambda \leq|\bigcup A|$.

ProOF. For (b), suppose $A=\left\{A_{\alpha} ; \alpha<\lambda\right\}$ is such a family, satisfying the $\kappa$-chain condition. For a contradiction, suppose $|\bigcup A|=\mu$ where $\mu<\lambda$, so $\kappa<\mu^{+} \leq \lambda$. We consider two cases.

Case 1. $c f(\mu)<\kappa$. Write $\bigcup A=\bigcup\left\{X_{\sigma} ; \sigma<c f(\mu)\right\}$ where alway $\left|X_{\sigma}\right|<\mu$. For each $A$ in $A$ there must be $\sigma(A)<c f(\mu)$ such that $\left|A \cap X_{\sigma(A)}\right|=\kappa$, and there must be $\sigma<c f(\mu)$ and $B \subseteq A$ with $B \mid \geq \mu^{+}$such that $\sigma(A)=\sigma$ for all $A \in B$. Since $A$ is almost disjoint, then $\left|\left\{A \cap X_{\sigma} ; A \in B\right\}\right|=|B| \geq \mu^{+}$which is impossible since $\left|\left[X_{\sigma}\right]^{\kappa}\right| \leq\left|X_{\sigma}\right|^{+} \leq \mu$.

Case 2. $\kappa \leq c f(\mu)$. For sequences $s, t \in{ }^{<\kappa} \mu$, write $t<\cdot s$ if $t$ is an initial segment of $s$. For each sequence $s \in{ }^{<\kappa} \mu$ we define an ordinal $\alpha(s)<\mu^{+}$ and, provided the length of $s$ is a successor ordinal, an element $x(s) \in \bigcup A$, by recursion on the length of $s$ as follows. Put $\bigcup A-\bigcup\left\{A_{\alpha(t)} ; t<\cdot s\right\}=\left\{x\left(s^{-\gamma}\right) ; \gamma<\right.$ $\mu\}$, (noting that this set has cardinality $\mu$ since $A$ is almost disjoint), where by $\widehat{s \gamma}$ we mean that sequence extending $s$ by one place and having value $\gamma$ at its last place. If there is $\alpha \in \mu^{+}-\{\alpha(t) ; t<\cdot s\}$ such that $x(t) \in A_{\alpha}$ for all $t \leq \cdot s$ of successor length, then $\alpha(s)$ is to be the least such $\alpha$, and otherwise $\alpha(s)=0$.

By $G C H,\left.\right|^{<\kappa} \mu \mid=\mu$ and we can choose $\beta \in \mu^{+}-\left\{\alpha(s) ; s \in{ }^{<\kappa} \mu\right\}$. For every $s \in<\kappa \mu$, since $A$ is almost disjoint $\left|A_{\beta} \cap \bigcup\left\{A_{\alpha(t)} ; t<\cdot s\right\}\right|<\kappa$ so $A_{\beta} \cap\left(\bigcup \mathcal{A}-\bigcup\left\{A_{\alpha(t)} ; t<\cdot s\right\}\right)$ is non-empty. Hence there is $\gamma<\mu$ such that $x\left(s^{\wedge} \gamma\right) \in A_{\beta}$. This means we can define a sequence $r \in{ }^{\kappa} \mu$ by recursively defining $r(\delta)$ for each $\delta<\kappa$ to be the least $\gamma$ such that $x\left((r \mid \delta)^{\wedge} \gamma\right) \in A_{\beta}$, where by $r \mid \delta$ we mean the sequence $(r(\varepsilon) ; \varepsilon<\delta)$. Put $x_{\delta}=x(r \mid(2 \delta+1))$ and $\alpha(\delta)=$ $\alpha(r \mid(2 \delta+1))$. The definition of $\alpha(\sigma)$ ensures that $x_{\delta} \in A_{\alpha(\sigma)}$ whenever $\delta \leq \sigma<\kappa$, whereas the definition of $x_{\delta}$ ensures that $x_{\delta} \notin A_{\alpha(\sigma)}$ whenever $\sigma<\delta<\kappa$. Hence $A_{\alpha(\sigma)} \cap\left\{x_{\delta} ; \delta<\kappa\right\}=\left\{x_{\delta} ; \delta \leq \sigma\right\}$. Thus if $D=\left\{x_{\delta} ; \delta<\kappa\right\}$, we have $D \cap A_{\alpha(\sigma)} \subset D \cap A_{\alpha(\tau)}$ whenever $\sigma<\tau<\kappa$, contradicting that $A$ satisfies the $\kappa$-chain condition. 
The proof of (a) proceeds as Case 2 above, defining $\alpha(s)$ and $x(s)$ for sequences $s \in{ }^{<\omega} \mu$.

LEMMA 3.2. Let $\kappa$ be regular and suppose $A$ is an almost disjoint $(\lambda, \kappa)$ family.

(a) If $A$ satisfies the $\aleph_{0}$-chain condition, then for every $C \in[A] \geq \kappa$ there is $C^{*} \subseteq A$ with $C \subseteq C^{*}$ and $\left|C^{*}\right|=|C|$ such that

$$
\forall A \in \mathcal{A}\left(\left|A \cap \bigcup C^{*}\right|=\kappa \Rightarrow A \in C^{*}\right) .
$$

(b) $(G C H)$ If $A$ satisfies the $\kappa$-chain condition, then for every $C \in[A] \geq \kappa$ with cf $(|C|) \geq \kappa$ there is $C^{*} \subseteq A$ with $C \subseteq C^{*}$ and $\left|C^{*}\right|=|C|$ such that (11) holds.

Proof. Take $C \in[A]^{\mu}$ where $\kappa \leq \mu<\lambda$, and $c f(\mu) \geq \kappa$ if (b) holds. (Obviously if $\mu=\lambda$, we can take $C^{*}=A$.) To cover both (a) and (b) at once, put $\eta=\kappa_{0}$ if (a) holds, and $\eta=\kappa$ if (b) holds. Recursively define families $\mathcal{C}_{\alpha} \in[A]^{\mu}$ for $\alpha<\eta$ as follows. Put $Z_{\alpha}=\bigcup\left\{\bigcup C_{\beta} ; \beta<\alpha\right\}$, so $\left|Z_{\alpha}\right|=\mu$. For each $S \in\left[Z_{\alpha}\right]^{<\eta}$, choose $A(\alpha, S) \in A-\bigcup\left\{\mathcal{C}_{\beta} ; \beta<\alpha\right\}$ with $S \subseteq A(\alpha, S)$ if such a set $A(\alpha, S)$ exists; otherwise let $A(\alpha, S)$ be empty. Put $X_{\alpha}=\bigcup \mathcal{C} \cup$ $Z_{\alpha} \cup \bigcup\left\{A(\alpha, S) ; S \in\left[Z_{\alpha}\right]^{<\eta}\right\}$. Our assumptions ensure that $\mu^{<\eta}=\mu$, so that $\left|X_{\alpha}\right|=\mu$. Now define $\mathcal{C}_{\alpha}=\left\{A \in \mathcal{A} ;\left|A \cap X_{\alpha}\right|=\kappa\right\}$. Since $\left\{A \cap X_{\alpha} ; A \in \mathcal{C}_{\alpha}\right\}$ is an an almost disjoint decomposition of $X_{\alpha}$ satisfying the $\eta$-chain condition, by Lemma 3.1 we have $\left|\left\{A \cap X_{\alpha} ; A \in C_{\alpha}\right\}\right| \leq\left|X_{\alpha}\right|=\mu$, and hence since $A$ is almost disjoint we have $\left|C_{\alpha}\right| \leq \mu$, so $\left|C_{\alpha}\right|=\mu$. Note that the definition of $C_{\alpha}$ ensures that $C \subseteq C_{\alpha}$ and $\bigcup\left\{C_{\beta} ; \beta<\alpha\right\} \subseteq C_{\alpha}$. Also $Z_{\alpha} \subseteq X_{\alpha} \subseteq Z_{\alpha+1}$. Define $C^{*}=\left\{C_{\alpha} ; \alpha<\eta\right\}$ so $C \subseteq C^{*} \subseteq A$ and $\left|C^{*}\right|=\mu$.

We show $C^{*}$ has the required property. Take $A \in A$ such that $\left|A \cap \cup C^{*}\right|=$ $\kappa$. Now $\bigcup C^{*}=\bigcup\left\{Z_{\alpha} ; \alpha<\eta\right\}$, and we claim that there is $\delta<\eta$ such that $\left|A \cap Z_{\delta}\right|=\kappa$. If so, $\left|A \cap X_{\delta}\right|=\kappa$ so $A \in C_{\delta} \subseteq C^{*}$ and the proof would be complete. So for a contradiction, suppose $\left|A \cap Z_{\delta}\right|<\kappa$ for all $\delta<\eta$. There must then be an increasing sequence $(\alpha(\sigma) ; \sigma<\eta)$ such that $A \cap\left(Z_{\alpha(\sigma+1)}-Z_{\alpha(\sigma)}\right)$ is non-empty for each $\sigma<\eta$, and (by deleting every second term if necessary) we may in fact suppose $A \cap\left(Z_{\alpha(\sigma+1)}-Z_{\alpha(\sigma)+1}\right)$ is non-empty. Choose $x_{\sigma} \in$ $A \cap\left(Z_{\alpha(\sigma+1)}-Z_{\alpha(\sigma)+1}\right)$, and put $S_{\sigma}=\left\{x_{\tau} ; \tau \leq \sigma\right\}$. Then $S_{\sigma} \in\left[Z_{\alpha(\sigma+1)}\right]^{<\eta}$. Now $S_{\sigma} \subseteq A$ and $A \notin \bigcup\left\{C_{\beta} ; \beta<\alpha(\sigma+1)\right\}$ so $S_{\sigma} \subseteq A\left(\alpha(\sigma+1), S_{\sigma}\right) \in A$. Put $A_{\sigma}=A\left(\alpha(\sigma+1), S_{\sigma}\right)$. Then $A_{\sigma} \subseteq X_{\alpha(\sigma+1)} \subseteq Z_{\alpha(\sigma+1)+1}$. If $\sigma<\tau<\kappa$ then $\alpha(\sigma+1) \leq \alpha(\tau)<\alpha(\tau)+1$ and $x_{\tau} \notin Z_{\alpha(\tau)+1}$ so $x_{\tau} \notin Z_{\alpha(\sigma+1)+1}$, and hence $x_{\tau} \notin A_{\sigma}$. Thus $A_{\sigma} \cap\left\{x_{\tau} ; \tau<\eta\right\}=\left\{x_{\tau} ; \tau \leq \sigma\right\}$. Put $D=\left\{x_{\tau} ; \tau<\eta\right\}$, so $D \cap A_{\sigma} \subset D \cap A_{\tau}$ whenever $\sigma<\tau<\eta$, contradicting that $\mathcal{A}$ satisfies the $\eta$-chain condition. 
THEOREM 3.3. Let $\kappa$ be regular and suppose $A$ is an almost disjoint $(\lambda, \kappa)$ family. Suppose either

(a) A satisfies the $\aleph_{0}$-chain condition, or

(b) A satisfies the $\kappa$-chain condition and $\lambda \leq \kappa^{\omega+}$.

Then $A$ has a $\kappa$-ordering.

PROOF. If $\lambda \leq \kappa$, then $A$ has a $\kappa$-ordering since $A$ is almost disjoint. For $\lambda>\kappa$ we proceed by induction on $\lambda$. Let $\left(\lambda_{\sigma} ; \sigma<c f(\lambda)\right)$ be a $\lambda$-sequence of cardinals with $\kappa \leq \lambda_{0} \leq \lambda_{1} \leq \cdots$ and $\sum\left(\lambda_{\sigma} ; \sigma<\tau\right) \leq \lambda_{\tau}$ for each $\tau<c f(\lambda)$, with always $c f\left(\lambda_{\sigma}\right) \geq \kappa$ if (b) holds. (Note such a sequence can be found except when $\lambda=\mu^{+}$where $c f(\mu)<\kappa$.) Put $\eta=\kappa_{0}$ if (a) holds, and $\eta=\kappa$ if (b) holds. Take an almost disjoint $(\lambda, \kappa)$-family $A$ with the $\eta$-chain condition. Write $A=\bigcup\left\{A_{\sigma} ; \sigma<c f(\lambda)\right\}$ where always $\left|A_{\sigma}\right|=\lambda_{\sigma}$. Use Lemma 3.2 to define families $B_{\sigma} \subseteq A$ for $\sigma<c f(\lambda)$ by $B_{\sigma}=\left(A_{\sigma} \cup \cup\left\{B_{\tau} ; \tau<\sigma\right\}\right)^{*}$, and it follows from Lemma 3.2 that always $\left|B_{\sigma}\right|=\lambda_{\sigma}$. Thus $B_{\rho} \subseteq \beta_{\sigma}$ if $\rho<\sigma<c f(\lambda)$, and $A=\bigcup\left\{B_{\sigma}, \sigma<c f(\lambda)\right\}$.

We proceed as in the proof of Lemma 1.3. For each $A$ let $\sigma(A)$ be the least $\sigma$ such that $A \in B_{\sigma}$. By the inductive hypothesis, there is a $\kappa$-ordering $\prec_{\sigma}$ of $B_{\sigma}$. Define $\prec$ on $A$ by

$$
A \prec B \Leftrightarrow \sigma(A)<\sigma(B) \text { or }\left[\sigma(A)=\sigma(B) \text { and } A \prec_{\sigma(A)} B\right] \text {. }
$$

Just as in the proof of Lemma 1.3 , this will be a $\kappa$-ordering of $A$ provided that

$$
\left|A \cap \bigcup\left\{\bigcup B_{\tau} ; \tau<\sigma(A)\right\}\right|<\kappa .
$$

If $\tau<\sigma(A)$ then $A \notin B_{\tau}=C^{*}$ where $C=A_{\tau} \cup \bigcup\left\{B_{\rho} ; \rho<\tau\right\}$, so by (11) we have $\left|A \cap \bigcup B_{\tau}\right|<\kappa$. So if $c f(\lambda) \leq \kappa$, certainly (12) holds. And if $\sigma(A)$ is a successor ordinal, $\sigma(A)=\xi+1$, then $\bigcup\left\{\bigcup B_{\tau} ; \tau<\sigma(A)\right\}=\bigcup B_{\xi}$ so there is no difficulty. We are left with the case that $\sigma(A)$ is a limit ordinal (and $\kappa<c f(\lambda)$, though we won't make use of this condition). Suppose for a contradiction that (12) is false, so $\left|A \cap \bigcup\left\{\bigcup B_{\tau} ; \tau<\sigma(A)\right\}\right|=\kappa$. Since $\bigcup B_{\rho} \subseteq \bigcup B_{\tau}$ if $\rho<\tau<\sigma(A)$, there must be an increasing sequence $(\rho(\sigma) ; \sigma<\kappa)$ of ordinals below $\sigma(A)$ such that $A \cap\left(\bigcup B_{\rho(\sigma+1)}-\bigcup B_{\rho(\sigma)}\right)$ is non-empty for each $\sigma<\kappa$. Choose $x_{\sigma} \in$ $A \cap\left(\bigcup B_{\rho(\sigma+1)}-\bigcup B_{\rho(\sigma)}\right)$, and put $S_{\sigma}=\left\{x_{\tau} ; \tau \leq \sigma\right\}$, so $S_{\sigma} \subseteq \bigcup B_{\rho(\sigma+1)}$. Put $C=A_{\rho(\sigma+1)} \cup \bigcup\left\{B_{\tau} ; \tau<\rho(\sigma+1)\right\}$, so $B_{\rho(\sigma+1)}=C^{*}$, and $S_{\sigma} \subseteq \bigcup C^{*}$. Consider the construction of $C^{*}$ in the proof of Lemma 3.2. We have $C^{*}=\bigcup\left\{C_{\alpha} ; \alpha<\eta\right\}$ so $\bigcup C^{*}=\bigcup\left\{\bigcup C_{\alpha} ; \alpha<\eta\right\}$, and $U \mathcal{C}_{\beta} \subseteq U \mathcal{C}_{\alpha}$ whenever $\beta<\alpha<\eta$. When $\sigma<\eta$ we have $S_{\sigma} \in\left[\cup C^{*}\right]^{<\eta}$ so there must be $\alpha<\eta$ such that $S_{\sigma} \subseteq \cup C_{\alpha}$. Thus $S_{\sigma} \in\left[Z_{\alpha+1}\right]^{<\eta}$ and so $S_{\sigma} \subseteq A\left(\alpha+1, S_{\sigma}\right)$ and $A\left(\alpha+1, S_{\sigma}\right) \in C^{*}$. (Note that $A\left(\alpha+1, S_{\sigma}\right)$ can't be empty since $S_{\sigma} \subseteq A$ and $A \notin \backslash J\left\{C_{\beta} ; \beta<\alpha\right\}$ because $A \notin B_{\rho(\sigma+1)}=C^{*}$.) Put $A_{\sigma}=A\left(\alpha+1, S_{\sigma}\right)$. Thus for $\sigma<\eta$, we have $A_{\sigma} \in$ $B_{\rho(\sigma+1)}$ with $S_{\sigma} \subseteq A_{\sigma}$. And if $\sigma<\tau<\eta$ then $x_{\tau} \notin \bigcup B_{\rho(\tau)}$ so $x_{\tau} \notin \bigcup B_{\rho(\sigma+1)}$ 
and hence $x_{\tau} \notin A_{\sigma}$. Thus $A_{\sigma} \cap\left\{x_{\tau} ; \tau<\eta\right\}=\left\{x_{\tau} ; \tau \leq \sigma\right\}$. Put $D=\left\{x_{\tau} ; \tau<\eta\right\}$, so $D \cap A_{\sigma} \subset D \cap A_{\tau}$ whenever $\sigma<\tau<\eta$, contradicting that $A$ satisfies the $\eta$-chain condition. Hence (12) holds, and the proof is complete.

The transfinite induction in Theorem 3.3(b) breaks down first when $\lambda=$ $\kappa^{(\omega+1)+}$. As was the case with Theorem 2.4(b) we can continue the induction if $\square_{\mu}$ holds for appropriate $\mu$.

THEOREM 3.4 $(V=L)$. Let $\kappa$ be regular and suppose $A$ is an almost disjoint $(\lambda, \kappa)$-family satisfying the $\kappa$-chain condition. Then $A$ has a $\kappa$-ordering.

Proof. We proceed by transfinite induction on $\lambda$, as in the proof of Theorem 3.3(b). The previous argument works unless $\aleph_{0}<\kappa<\lambda=\mu^{+}$and $c f(\mu)<\kappa$, so suppose this to be the case. Let $A=\left\{A_{\delta} ; \delta<\mu^{+}\right\}$, where we may suppose $\bigcup A=\mu^{+}$. We have the sets $D(\alpha)$ and $T(\alpha, \sigma)$ for $\alpha<\mu^{+}$and $\sigma<c f(\mu)$ as in Lemma 2.5. Define families $\left.B_{\varepsilon} \in[A]\right]^{\leq \mu}$ and limit ordinals $l_{\varepsilon}$ with $\mu \leq l_{\varepsilon}<\mu^{+}$ by transfinite recursion for $\varepsilon<\mu^{+}$as follows. Put $B_{0}=\left\{A_{0}\right\}$ and let $l_{0}$ be the least limit ordinal $\gamma>\bigcup A_{0}$. Suppose $B_{\delta}$ defined for all $\delta<\varepsilon$ (for $\varepsilon>0$ ). If $\varepsilon$ is a limit ordinal, define $l_{\varepsilon}=\bigcup\left\{l_{\delta} ; \delta<\varepsilon\right\}$. If $\varepsilon$ is a successor, define $l_{\varepsilon}$ to be the least ordinal $\gamma>l_{\varepsilon-1} \cup \bigcup\left\{B_{\delta} ; \delta<\varepsilon\right\}$. So $l_{\varepsilon}<\mu^{+}$. For each limit $\alpha<l_{\varepsilon}$ and for each $\sigma<c f(\mu)$, for each $S \in[T(\alpha, \sigma)]^{<\kappa}$ choose $A(\varepsilon, \alpha, \sigma, S) \in A-\bigcup\left\{B_{\delta} ; \delta<\varepsilon\right\}$ with $S \subseteq A(\varepsilon, \alpha, \sigma, S)$ if such a set $A(\varepsilon, \alpha, \sigma, S)$ exists; otherwise let $A(\varepsilon, \alpha, \sigma, S)$ be empty. Put

$$
X_{\varepsilon}=l_{\varepsilon} \cup A_{\varepsilon} \cup \bigcup\left\{A(\varepsilon, \alpha, \sigma, S) ; \exists \text { limit } \alpha<l_{\varepsilon} \exists \sigma<c f(\mu)\left(S \in[T(\alpha, \sigma)]^{<\kappa}\right)\right\}
$$

and finally put

$$
B_{\varepsilon}=\left\{A \in A ;\left|A \cap X_{\varepsilon}\right|=\kappa\right\} .
$$

We have to check that $\left|B_{\varepsilon}\right| \leq \mu$. Note if $\delta<\varepsilon$ then $\bigcup B_{\delta} \subseteq l_{\varepsilon} \subseteq X_{\varepsilon}$, so $B_{\delta} \subseteq B_{\varepsilon}$. Always $|T(\alpha, \sigma)|<\mu$, so $\left|[T(\alpha, \sigma)]^{<\kappa}\right| \leq \mu$ and hence $\left|X_{\varepsilon}\right| \leq \mu$. Since $\left\{A \cap X_{\varepsilon} ; A \in B_{\varepsilon}\right\}$ is an almost disjoint decomposition of $X_{\varepsilon}$ with the $\kappa$-chain condition, $\left|\left\{A \cap X_{\varepsilon} ; A \in B_{\varepsilon}\right\}\right| \leq \mu$ by Lemma 3.1. Hence, since $A$ is almost disjoint, we have $\left|B_{\varepsilon}\right| \leq \mu$.

For $A \in \mathcal{A}$, define $\varepsilon(A)$ to be the least $\varepsilon$ such that $\left|A \cap l_{\varepsilon}\right|=\kappa$. (Such $\varepsilon(A)$ exists, for if $A=A_{\delta}$ we have $A \in B_{\delta}$ so $A \subseteq \bigcup B_{\delta} \subseteq l_{\delta+1}$.) We claim that $\varepsilon(A)$ is not a limit ordinal. For suppose on the contrary that $\varepsilon(A)$ is a limit. Write $\xi=l_{\varepsilon(A)}$, so $\xi=\bigcup\left\{l_{\delta} ; \delta<\varepsilon(A)\right\}$ and $c f(\xi)=c f(\varepsilon(A))$ since the $l_{\delta}$ increase with $\delta$. There must be an increasing sequence $(\delta(\sigma) ; \sigma<c f(\xi))$ with $\delta(\sigma)<\varepsilon(A)$ such that $\left\{l_{\delta(\sigma)} ; \sigma<c f(\xi)\right\}$ is cofinal in $\xi$. Since $\left|A \cap l_{\delta}\right|<\kappa$ for $\delta<\varepsilon(A)$, yet $|A \cap \xi|=\kappa$, we must have $c f(\xi)=\kappa$ and we may suppose $A \cap\left(l_{\delta(\sigma+1)}-l_{\delta(\sigma)+1}\right)$ is non-empty for each $\sigma<\kappa$. Also since $c f(\xi)=\kappa>\omega$, we have $D(\xi)$ cofinal in $\xi$. Define recursively $\delta(\sigma) \in D(\xi)$ and $\rho(\sigma)<\varepsilon(A)$ for $\sigma<\kappa$ as follows. Let $\gamma(0)$ be the least element of $D(\xi)$, and if $\sigma$ is a limit let $\gamma(\sigma)$ 
be the least $\gamma$ in $D(\xi)$ with $\gamma \geq \bigcup\{\gamma(\tau) ; \tau<\sigma\}$. Suppose $\gamma(\sigma)$ is defined. Let $\rho(\sigma)$ be the least $\rho<\varepsilon(A)$ such that $\gamma(\sigma)<l_{\delta(\rho)}$, and then define $\gamma(\sigma+1)$ to be the least $\gamma$ in $D(\xi)$ with $\gamma>l_{\delta(\rho(\sigma)+1)}$. Choose $x_{\sigma} \in A \cap\left(l_{\delta(\rho(\sigma)+1)}-l_{\delta(\rho(\sigma))+1}\right)$, so $x_{\sigma} \in A \cap(\gamma(\sigma+1)-\gamma(\sigma))$ since $\gamma(\sigma+1)>l_{\delta(\rho(\sigma)+1)}$ and $\gamma(\sigma)<l_{\delta(\rho(\sigma))}$. Now $\gamma(\sigma+1)=\bigcup\{T(\gamma(\sigma+1), \zeta) ; \zeta<c f(\mu)\}$ so there is $\zeta(\sigma)<c f(\mu)$ such that $x_{\sigma} \in T(\gamma(\sigma+1), \varsigma(\sigma))$. Because $c f(\mu)<\kappa$, there are $H \in[\kappa]^{\kappa}$ and $\varsigma<c f(\mu)$ such tht $\varsigma(\sigma)=\varsigma$ for all $\sigma \in H$. By re-indexing, we may suppose $\varsigma(\sigma)=\varsigma$ for all $\sigma<\kappa$. For each $\sigma<\kappa$, put $S_{\sigma}=\left\{x_{\tau} ; \tau<\sigma\right\}$. Since all $\gamma(\sigma) \in D(\xi)$, from Lemma $2.5 T(\gamma(\tau+1), \varsigma) \subseteq T(\gamma(\sigma), \varsigma)$ whenever $\tau<\sigma<\kappa$, so $S_{\sigma} \subseteq T(\gamma(\sigma), \varsigma)$. Put $A_{\sigma}=A\left(\delta(\rho(\sigma)), \gamma(\sigma), \varsigma, S_{\sigma}\right)$. Note $S_{\sigma} \subseteq A_{\sigma} \in \mathcal{A}$, since $S_{\sigma} \subseteq A$ and $A \notin \bigcup\left\{B_{\delta} ; \delta<\delta(\rho(\sigma))\right\}$ (for if $A \in B_{\delta}$ then $A \subseteq l_{\delta+1}$ so $\left|A \cap l_{\delta+1}\right|=\kappa$, yet $\left.\delta(\rho(\sigma))<\varepsilon(A)\right)$. Thus $A_{\sigma} \subseteq X_{\delta(\rho(\sigma))}$, so $A_{\sigma} \in B_{\delta(\rho(\sigma))}$, and hence $A_{\sigma} \subseteq l_{\delta(\rho(\sigma))+1}$. And if $\tau \leq \sigma$ then $x_{\sigma} \notin l_{\delta(\rho(\tau))+1}$ so $x_{\sigma} \notin A_{\tau}$. Thus $A_{\sigma} \cap\left\{x_{\tau} ; \tau<\kappa\right\}=S_{\sigma}=\left\{x_{\tau} ; \tau<\sigma\right\}$. Put $D=\left\{x_{\tau} ; \tau<\kappa\right\}$, so $D \cap A_{\sigma} \subset D \cap A_{\tau}$ whenever $\sigma<\tau<\kappa$ contradicting that $A$ satisfies the $\kappa$-chain condition. This establishes our claim that $\varepsilon(A)$ is not a limit ordinal.

We complete the proof that $A$ has a $\kappa$-ordering by appealing to Lemma 1.4. Every $B$ in $[A]^{<\lambda}$ has a $\kappa$-ordering by the inductive hypothesis, so (i) of Lemma 1.4 holds. For (ii), define $U_{\rho}=l_{\rho}$, for $\rho<\mu^{+}=c f(\lambda)$. Certainly $\bigcup A=\mu^{+}=$ $\bigcup\left\{U_{\rho} ; \rho<\mu^{+}\right\}$and $U_{\rho} \subseteq U_{\tau}$ if $\rho<\tau<\mu^{+}$. Take any $A \in A$. Since $\varepsilon(A)$ is not a limit, we can put $\rho=\varepsilon(A)-1$. Now $\left|A \cap l_{\varepsilon(A)}\right|=\kappa$, so $A \in B_{\varepsilon(A)}$ and hence $A \subseteq l_{\varepsilon(A)+1}=U_{\rho+2}$. Since $\rho<\varepsilon(A)$ we have $\left|A \cap U_{\rho}\right|<\kappa$. Hence (iia) holds. To verify that (iib) holds, suppose $A \subseteq U_{\rho}=l_{\rho}$. Then $A \in B_{\rho}$. Since $\left|B_{\rho}\right| \leq \mu$, this means that (iib) holds. Hence by Lemma 1.4, $A$ has a $\kappa$-ordering.

\section{Applications}

In this section we present several applications of the idea of a $\theta$-ordering. The first is a trivial observation, but when combined with Theorems 2.4 and 2.6, it gives a proof of Komjáth's theorem mentioned in the introduction ([5], Theorem $5)$.

THEOREM 4.1. If $A$ is a $(\lambda, \kappa)$-family which possesses a $\kappa$-ordering, then $A$ is sparse.

PROOF. Let $\prec$ be a $\kappa$-ordering of $A$. Define $f: A \rightarrow P \cup A$ by

$$
f(A)=A \cap \bigcup\{B \in A ; B \prec A\} .
$$

By $(3), f(A) \in[A]^{<\kappa}$ and clearly $\{A-f(A) ; A \in \mathcal{A}\}$ is a pairwise disjoint family, so $f$ shows $A$ is sparse. 
The next couple of results concern transversals of the family $\mathcal{A}$. The case $\kappa=\aleph_{0}$ of Theorem 4.2 is essentially due to Davies and Erdös ([1], Proposition A), and their construction carries over to larger $\kappa$.

THEOREM 4.2. Every $(\lambda, \kappa)$-family $A$ which possesses a $\kappa$-ordering can be split into $\kappa$ subfamilies, $A=\bigcup\left\{A_{\xi} ; \xi<\kappa\right\}$, where each subfamily $A_{\xi}$ has a 2-transversal $T_{\xi}$, and moreover $\bigcup A=\bigcup\left\{T_{\xi} ; \xi<\kappa\right\}$.

ProOF. Let $\prec$ be a $\kappa$-ordering of $A$. For each $A \in A$, write $A-\bigcup\{B \in$ $A ; B \prec A\}=\{a(A, \alpha) ; \alpha<\kappa\}$, where $a(A, \alpha) \neq a(A, \beta)$ if $\alpha \neq \beta$. By transfinite recursion on $\prec$, for each $A \in A$ use induction to choose $\xi(A, \alpha)$ for $\alpha<\kappa$ so that $\xi(A, \alpha) \in \kappa-(\{\xi(B, \beta) ; B<A$ and $\beta<\kappa$ and $a(B, \beta) \in A\} \cup\{\xi(A, \gamma) ; \gamma<\alpha\})$. (Since $|A \cap \bigcup\{B ; B \prec A\}|<\kappa$ and $a(B, \beta) \neq a(C, \gamma)$ if $(B, \beta) \neq(C, \gamma)$, such a choice is possible.) For each $\xi<\kappa$, put

$$
\begin{gathered}
T_{\xi}=\{a(A, \alpha) ; \xi(A, \alpha)=\xi\}, \text { and } \\
A_{\xi}=\{A \in \mathcal{A} ; \xi(A, \alpha)=\xi, \text { for some } \alpha<\kappa\} .
\end{gathered}
$$

Clearly $A=\bigcup\left\{A_{\xi} ; \xi<\kappa\right\}$ and $\bigcup \mathcal{A}=\bigcup\left\{T_{\xi} ; \xi<\kappa\right\}$. We show that $\left|T_{\xi} \cap A\right|=1$ for each $A$ in $A_{\xi}$, so $T_{\xi}$ is a 2-transversal of $A_{\xi}$. If $A \in A_{\xi}$, then $a(A, \alpha) \in A \cap T_{\xi}$ for that $\alpha$ with $\xi(A, \alpha)=\xi$. Take any $x \in A \cap T_{\xi}$ with $x \neq a(A, \alpha)$ for this $\alpha$. Then either (i) $x=a(B, \beta)$ and $\xi(B, \beta)=\xi$ for some $B$ with $B \prec A$, which is contrary to the choice of $\xi(A, \alpha)$, or (ii) $x=a(B, \beta)$ and $\xi(B, \beta)=\xi$ for some $B$ with $A \prec B$, which is contrary to the choice of $\xi(B, \alpha)$, or (iii) $x=a(A, \beta)$ and $\xi(A, \beta)=\xi$ for some $\beta \neq \alpha$, contrary to $\xi(A, \alpha)=\xi$. Hence there is no such $x$, and thus $\left|A \cap T_{\xi}\right|=1$ as required.

THEOREM 4.3. Let $A$ be a $(\lambda, \kappa)$-family with a $\theta$-ordering, and suppose for every subfamily $B \in[A]^{\kappa}$ the family $\{B-R(B) ; B \in B\}$ has a $\theta$-transversal, for every choice of $R(B) \in[B]^{<\theta}$. Then $A$ has $\theta$-transversal.

ProOF. Let $\prec$ be a $\theta$-ordering of $\mathcal{A}$, with family of intervals $I$. For each $I \in I$, put $I^{*}=\bigcup I-\bigcup\{\bigcup J ; J \in I$ and $J \prec I\}$. Take $A \in A$, and suppose $A \in I$. Put $R(A)=A \cap \bigcup\{\bigcup J ; J \in I$ and $J \prec I\}$ so $R(A) \in[A]^{<\theta}$ by (1), and $A \cap I^{*}=A-R(A)$. Since $|I| \leq \kappa$, by assumption there is a $\theta$-transversal, say $T(I)$, for $\left\{A \cap I^{*} ; A \in I\right\}$, and we may assume $T(I) \subseteq I^{*}$. But then $T=\bigcup\{T(I) ; I \in I\}$ is a $\theta$-transversal for $A$, since for each $A$ in $\mathcal{A}$, if $A \in I$ then $A=\left(A \cap I^{*}\right) \cup R(A)$ so $A \cap T \subseteq\left(A \cap I^{*} \cap T(I)\right) \cup R(A)$, and consequently $1 \leq|A \cap T|<\theta$ as required.

Combining Theorems 3.3 and 4.3, together with the observation that for regular $\kappa$, every almost disjoint $(\kappa, \kappa)$-family has a $\kappa$-transversal provides a proof of ([8], Theorem 3.2) (which can be extended by using Theorem 3.4 as well). 
Results on the existence of $\theta$-transversals for families $A$ when $A$ satisfies intersection conditions were first studied extensively by Erdös and Hajnal [2]. (Having a $\theta$-transversal was there referred to as possessing property $B(\theta)$.) We can deduce their results from Theorem 4.3 , as follows.

COROLlaRY 4.4. Let $A$ be an almost disjoint $(\lambda, \kappa)$-family. Take $\theta<\kappa$ and suppose either $A$ satisfies $C(2, \theta)$, or else $\kappa$ is regular and $A$ satisfies $C(\kappa, \theta)$. Then

(a) (GCH) A has a $\theta^{++}$-transversal.

(b) $(G C H)$ Suppose either $\theta^{+}=\kappa$ or else $\theta^{+}<\kappa$ but $\kappa \neq \mu^{+}$where $c f(\theta)=$ $c f(\mu)$, and suppose $c f(\eta) \neq c f(\theta)$ whenever $\kappa \leq \eta<\lambda$. Then $A$ has a $\theta^{+}$. transversal.

(c) $(V=L)$ A has a $\theta^{+}$-transversal.

PROOF. The result will follow from Theorems 4.3, 2.4 and 2.6 once we show that every $(\kappa, \kappa)$-family $B$ satisfying these conditions has a $\theta^{++}$-transversal or a $\theta^{+}$-transversal, respectively. For (b), take the appropriate $(\kappa, \kappa)$-family $B=$ $\left\{B_{\alpha} ; \alpha<\kappa\right\}$ and we show that $B$ has a $\theta^{+}$-transversal. (This is essentially ([2], Result 4.9).) If $\theta^{+}=\kappa$, the result is immediate since $\kappa$ is regular and $A$ is almost disjoint. So suppose $\theta^{+}<\kappa$. Recursively define elements $x_{\beta} \in \cup B$ for $\beta<\kappa$, as follows. Choose $x_{0} \in B_{0}$. For $\beta>0$, put $X_{\beta}=\left\{x_{\gamma} ; \gamma<\beta\right\}$ and let $C_{\beta}=\left\{B \in B ;\left|B \cap X_{\beta}\right| \geq \theta\right\}$. Choose $x_{\beta} \in B_{\beta}-\bigcup C_{\beta}$ if $B_{\beta}-\bigcup C_{\beta}$ is non-empty, and otherwise put $x_{\beta}=x_{0}$.

We claim that $\left|C_{\beta}\right|<\kappa$ for all $\beta<\kappa$. Certainly $\left|\left\{B \cap X_{\beta} ; B \in C_{\beta}\right\}\right|<\kappa$, for this is immediate if $\left|X_{\beta}\right|^{+}<\kappa$, and if $\left|X_{\beta}\right|^{+}=\kappa$ it follows from Lemma 2.1 since in this case $\left\{B \cap X_{\beta} ; B \in \mathcal{C}_{\beta}\right\}$ satisfies $C\left(\left|X_{\beta}\right|^{+}, \theta\right)$ and $c f\left(\left|X_{\beta}\right|\right) \neq c f(\theta)$ by hypothesis. Also, for any $Z \in\left[X_{\beta}\right]^{\theta}$, we have $\left|\left\{B \in B ; Z \subseteq B \cap X_{\beta}\right\}\right|<\kappa$ since $B$ satisfies $C(\kappa, \theta)$, and in fact $\left|\left\{B \in B ; Z \subseteq B \cap X_{\beta}\right\}\right|<2$ if $\kappa$ is singular since then $B$ satisfies $C(2, \theta)$. Hence $\left|C_{\beta}\right|<\kappa$. Thus, if $B_{\beta} \notin C_{\beta}$ then $\left|B_{\beta}-\bigcup C_{\beta}\right|=\kappa$, and so then $x_{\beta} \in B_{\beta}$.

Put $T=\left\{x_{\beta} ; \beta<\kappa\right\}$, so always $\left|T \cap B_{\beta}\right| \geq 1$. And if for any $B \in B$ we have $\left|B \cap X_{\beta}\right|=\theta$ then for all $\gamma \geq \beta$ it follows that $B \in C_{\gamma}$ so either $x_{\gamma}=x_{0}$ or $x_{\gamma} \notin B$, and hence $|T \cap B|=\theta$. Thus for all $B \in B$ we have $1 \leq|T \cap B| \leq \theta$, so $T$ is a $\theta^{+}$-transversal of $B$.

The argument for (a) is similar, putting $\mathcal{C}_{\beta}=\left\{B \in B ;\left|B \cap X_{\beta}\right|>\theta\right\}$.

Case (b) also covers case (c), except when $\kappa=\mu^{+}>\theta^{+}$where $c f(\mu)=\operatorname{cf}(\theta)$. For this situation we use $\square_{\mu}$. As in case (b), write $B=\left\{B_{\alpha} ; \alpha<\kappa\right\}$, and we may suppose $\bigcup B=\mu^{+}$. We have the sets $D(\alpha)$ and $T(\alpha, \sigma)$ for $\alpha<\mu^{+}$and $\sigma<c f(\mu)$, as in Lemma 2.5. Recursively define elements $x_{\beta} \in \bigcup B$ for $\beta<\kappa$, ensuring that $x_{\gamma}<x_{\beta}$ whenever $\gamma<\beta$ (unless $x_{\beta}=x_{0}$ ). Choose $x_{0} \in B_{0}$. For $\beta>0$, put $X_{\beta}=\left\{x_{\gamma} ; \gamma<\beta\right\}$ and let $l_{\beta}$ be the least ordinal $\xi$ with $c f(\xi)>\omega$ 
and $\xi>\bigcup X_{\beta}$. Define

$$
C_{\beta}=\left\{B \in B ; \exists \text { limit } \alpha<l_{\beta} \exists \sigma<c f(\mu)\left(\left|B \cap X_{\beta} \cap T(\alpha, \sigma)\right| \geq \theta\right)\right\} .
$$

Since $\left|[T(\alpha, \sigma)]^{\theta}\right| \leq \mu$ and $|\{B \in B ; B \cap T(\alpha, \sigma)=X\}|<\kappa$ for each $X \in$ $[T(\alpha, \sigma)]^{\theta}$, we have $\left|C_{\beta}\right| \leq \mu$. Hence if $B_{\beta} \notin C_{\beta}$ then $\left|B_{\beta}-\bigcup C_{\beta}\right|=\kappa$ since $B$ is almost disjoint, and we can choose $x_{\beta} \in B_{\beta}-\bigcup \mathcal{C}_{\beta}$ with $x_{\beta}>x_{\gamma}$ for all $\gamma<\beta$. If $B_{\beta} \in C_{\beta}$, put $x_{\beta}=x_{0}$.

Put $T=\left\{x_{\beta} ; \beta<\kappa\right\}$, so always $\left|B_{\beta} \cap T\right| \geq 1$. We claim that $|B \cap T| \leq \theta$ for each $B \in B$, so $T$ is a $\theta^{+}$-transversal of $B$. Suppose for a contradiction that there is $B \in B$ with $|B \cap T| \geq \theta^{+}$. There must be $\delta<\kappa$ such that $\left|B \cap X_{\delta}\right| \geq \theta^{+}$, for otherwise we could choose $\beta(\xi)$ for $\xi<\theta^{+}$such that $\left\{x_{\beta(\xi)} ; \xi<\theta^{+}\right\}$was cofinal in $B$, and hence in $\kappa$ (since $B \in[\kappa]^{\kappa}$ ), which is impossible with $c f(\kappa)=\mu^{+}>\theta^{+}$. Hence $\left|B \cap X_{\delta} \cap l_{\delta}\right| \geq \theta^{+}$. Since $l_{\delta}=\bigcup\left\{T\left(l_{\delta} ; \sigma\right) ; \sigma<c f(\mu)\right\}$ and $c f(\mu)=$ $c f(\theta)<\theta^{+}$there must be $\sigma<c f(\mu)$ such that $\left|B \cap X_{\delta} \cap T\left(l_{\delta}, \sigma\right)\right| \geq \theta^{+}$. And $T\left(l_{\delta}, \sigma\right)=\bigcup\left\{T(\beta, \sigma) ; \beta \in D\left(l_{\delta}\right)\right\}$ with $T(\gamma, \sigma) \subseteq T(\beta, \sigma)$ whenever $\beta, \gamma \in D\left(l_{\delta}\right)$ with $\gamma<\beta$, so there must be $\beta \in D\left(l_{\delta}\right)$ such that $\left|B \cap X_{\delta} \cap T(\beta, \sigma)\right| \geq \theta$. Let $\beta^{*}$ be the least limit ordinal such that $\exists \sigma<c f(\mu)\left(\left|B \cap X_{\delta} \cap T\left(\beta^{*}, \sigma\right)\right|=\theta\right)$. Let $\gamma$ be least such that $x_{\gamma} \geq \beta^{*}$, so $\left|B \cap X_{\gamma} \cap T\left(\beta^{*}, \sigma\right)\right|=\theta$. Also $B \in \mathcal{C}_{\beta}$ whenever $\beta>\gamma$, since then $X_{\gamma} \subseteq X_{\beta}$ and $\beta^{*} \leq x_{\gamma} \leq l_{\gamma+1} \leq l_{\beta}$, so that $x_{\beta} \notin B$ unless $x_{\beta}=x_{0}$. Hence $B \cap T \subseteq X_{\gamma}$. By the choice of $\beta^{*}$, we have $\left|B \cap X_{\gamma} \cap T\left(\beta^{*}, \tau\right)\right| \leq \theta$ for all $\tau<c f(\mu)$ and hence $\left|B \cap X_{\gamma}\right| \leq \theta$ since $B \cap X_{\gamma} \subseteq \beta^{*}=\bigcup\left\{T\left(\beta^{*}, \tau\right) ; \tau<c f(\mu)\right\}$. Hence $|B \cap T| \leq \theta$, contradicting that $|B \cap T| \geq \theta^{+}$. Thus $T$ is a $\theta^{+}$-transversal of $B$, and the proof is complete.

The final result concerns the existence of $\Delta$-families. The family $B$ is said to be a $\Delta$-family if there is a fixed set $Z$ such that $B \cap C=Z$ for all distinct $B, C \in B$.

THEOREM 4.5. Suppose $\kappa^{<\kappa}=\kappa$, and let $\mathcal{A}$ be a $\left(\kappa^{+}, \kappa\right)$-family which possesses a $\kappa$-ordering. Then there is a $\Delta$-family $B \subseteq A$ with $|B|=\kappa^{+}$.

ProOF. Let $\prec$ be a $\kappa$-ordering of $\mathcal{A}$, and we may suppose $\mathscr{A}=\left\{A_{\alpha} ; \alpha<\kappa^{+}\right\}$ is the enumeration of $A$ in increasing $\prec$-order, so always

$$
\left|A_{\alpha} \cap \bigcup\left\{A_{\beta} ; \beta<\alpha\right\}\right|<\kappa .
$$

Recursively choose subsets $X_{\gamma} \subseteq \kappa^{+}$(possibly empty) for $\gamma \leq \kappa$ as follows: put $X(\gamma)=\bigcup\left\{X_{\beta} ; \beta<\gamma\right\}$ and $A(\gamma)=\bigcup\left\{A_{\alpha} ; \alpha \in X(\gamma)\right\}$, and choose $X_{\gamma} \subseteq$ $\kappa^{+}-X(\gamma)$ maximal such that the family $\left\{A_{\alpha}-A(\gamma) ; \alpha \in X_{\gamma}\right\}$ is pairwise disjoint. We claim there is $\gamma<\kappa$ with $\left|X_{\gamma}\right|=\kappa^{+}$. For if not, $\left|X_{\beta}\right| \leq \kappa$ for all $\beta<\kappa$, and so $|X(\kappa)| \leq \kappa$. Take $\delta \in \kappa^{+}$with $\delta>\alpha$ for all $\alpha \in X(\kappa)$, then by the maximality 
of $X_{\beta}$, for each $\beta<\kappa$ there must be $\alpha(\beta) \in X_{\beta}$ such that $\left(A_{\delta}-A(\beta)\right) \cap\left(A_{\alpha(\beta)}-\right.$ $A(\beta))$ is non-empty, so we can choose $x_{\beta} \in\left(A_{\delta} \cap A_{\alpha(\beta)}\right)-A(\beta)$. Now $x_{\beta} \neq x_{\gamma}$ if $\gamma<\beta<\kappa$, since $x_{\gamma} \in A_{\alpha(\gamma)} \subseteq A(\beta)$. Since

$$
\left\{x_{\beta} ; \beta<\kappa\right\} \subseteq A_{\delta} \cap \bigcup\left\{A_{\alpha(\beta)} ; \beta<\kappa\right\} \subseteq A_{\delta} \cap \bigcup\left\{A_{\alpha} ; \alpha<\delta\right\},
$$

this contradicts (13), and proves the claim.

Let $\gamma$ be least such that $\left|X_{\gamma}\right|=\kappa^{+}$. Then $|X(\gamma)| \leq \kappa$ and $|A(\gamma)|=\kappa$, and if $X=\left\{\beta \in X_{\gamma} ; \forall \alpha \in X(\gamma)(\alpha<\beta)\right\}$ then $|X|=\kappa^{+}$. For $\beta \in X$, since $A_{\beta} \cap A(\gamma) \subseteq A_{\beta} \cap \bigcup\left\{A_{\alpha} ; \alpha<\beta\right\}$, by (13) $A_{\beta} \cap A(\gamma) \in[A(\gamma)]^{<\kappa}$. Since $\kappa^{<\kappa}=\kappa$, there must be $Z$ in $[A(\gamma)]^{<\kappa}$ and $Y \in[X]^{\kappa^{+}}$such that $A_{\beta} \cap A(\gamma)=Z$ for all $\beta \in Y$. However, $\left\{A_{\beta}-A(\gamma) ; \beta \in Y\right\}$ is pairwise disjoint, so $\left\{A_{\beta} ; \beta \in Y\right\}$ is a $\Delta$-family of size $\kappa^{+}$.

Combining Theorems 4.5 and 2.4 proves a result of Erdös, Milner and Rado ([4], Theorem 1), that for $\kappa$ regular, every almost disjoint $\left(\kappa^{+}, \kappa\right)$-family satisfying $C\left(\kappa^{+}, \theta\right)$ where $\theta<\kappa$ contains a $\Delta$-family of size $\kappa^{+}$. Combining Theorems 4.5 and 3.3 gives a result of Williams ([7], Corollary 2.9), that for $\kappa$ regular, every almost disjoint $\left(\kappa^{+}, \kappa\right)$-family with the $\kappa$-chain condition contains a $\Delta$-family of size $\kappa^{+}$.

\section{References}

[1] R. O. Davies and P. Erdös, 'Splitting almost-disjoint collections of sets into subcollections admitting almost-transversals,' Infinite and finite sets, Vol. I, edited by A. Hajnal, R. Rado and V. T. Sós, pp. 307-322 (North-Holland, 1975).

[2] P. Erdös and A. Hajnal, 'On a property of families of sets,' Acta Math. Acad. Sci. Hungar. 12 (1961), 87-123.

[3] P. Erdös and S. H. Hechler, 'On maximal almost-disjoint families over singular cardinals', Infinite and finite sets, Vol. I, edited by A. Hajnal, R. Rado and V. T. Sós, pp. 597-604 (North-Holland, 1975).

[4] P. Erdös, E. C. Milner and R. Rado, 'Intersection theorems for systems of sets (III)', J. Austral. Math. Soc. 18 (1974), 22-40.

[5] P. Komjâth, 'Families close to disjoint ones', Acta Math. Acad. Sci. Hungar. 43 (1984), 199-207.

[6] N. H. Williams, Combinatorial set theory (Studies in Logic and the Foundations of Mathematics, 91, North-Holland, 1977).

[7] N. H. Williams, 'Chain conditions and the existence of delta-families', Math. Proc. Cambridge Philos. Soc. 83 (1978), 167-179.

[8] N. H. Williams, 'Families of sets having property B', Period. Math. Hungar. 12 (1981), 181-190.

Department of Mathematics

University of Queensland

St. Lucia, Queensland 4067

Australia 